\title{
Ultrasonic evaluation of carotid artery elasticity in People Living with HIV
}

\author{
Zhao Ningbo $^{1 \#}$, Huang Xiulin² ${ }^{2 \#}$,Zeng Weimei ${ }^{2}$, Wang Fuxiang ${ }^{3}$, Dong Changfeng ${ }^{1 *}$, Feng Cheng $^{1 *}$
}

${ }^{1}$ Department of Ultrasound, Shenzhen Third People's Hospital, Shenzhen, Guangdong, China, 518112; ${ }^{2}$ Guangdong Medical University, Zhanjiang, Guangdong, 524000; Infection Department, Shenzhen Third People's Hospital, Shenzhen, Guangdong, China, 518112.

Dong Changfeng, Email:chaosheng-02@szsy.sustech.edu.cn, https://orcid.org/0000-0003-4236-9394.

Feng Cheng, Email:chaosheng-01@szsy.sustech.edu.cn, https://orcid.org/0000-0001-8154-6029.

\# Ningbo Zhao and Xiuling Huang contributed to this study equally.

${ }^{*}$ Changfeng Dong and Cheng Feng is the corresponding author.

Article History Received 20 May 2021 Accepted 25 May 2021 Published 30 June 2021

Cite this Article Zhao Ningbo, Huang Xiulin, Zeng Weimei, Wang Fuxiang, Dong Changfeng, Feng Cheng. Ultrasonic evaluation of carotid artery elasticity in People Living with HIV [J].Medical Research, 2021.3(2):1727, http://dx.doi.org/10.6913/MRHK.202106_3(2).0003

Copyright @ 2021 Creative Publishing Co., Limited. All rights reserved. Email:mrhk26640333@gmail.com

\section{ABSTRACT}

Objective To evaluate the quantitative parameters of carotid elasticity in healthy people and People Living With HIV(PLWH) by ultrasonic vascular elasticity quantitative technique, and to explore the feasibility of this technique in non-invasive evaluation of preclinical arterial elasticity in patients with PLWH atherosclerosis.

Methods Subjects with carotid intima-media thickness (IMT) $<1.5 \mathrm{~mm}$ were divided into three groups: healthy control group $(n=60)$, HIV baseline group $(n=291)$ and HAART group $(n=67)$ with HIV infection who had received highly active antiretroviral therapy ((HAART) for more than 5 years. Quantitative analysis of ultrasonic vascular elasticity was performed in all subjects, and the software automatically quantified carotid artery IMT, artery internal diameter, motion amplitude, vascular hardness coefficient and carotid artery pulse wave velocity, ( caPWV), and thean to compare the ultrasonic parameters and blood lipids of each group. In HAART group, the correlation between brachial-ankle pulse wave velocity (baPWV) measured by traditional pressure wave method and caPWV was analyzed.

Results Total Cholesterol (TC), triglyceride (TG) and high-density lipoprotein cholesterol (HDL-C) in HAART group were higher than those in baseline group $(\mathrm{P}<0.017)$. Hardness coefficient and caPWV of bilateral carotid arteries in HAART group were higher than those in baseline group $(\mathrm{P}<0.017)$, but the movement amplitude was lower than that in baseline group. In the healthy group, there was no significant difference between the left and right carotid arteries $(\mathrm{P}>0.05)$. In HAART group, baPWV was linearly correlated with caPWV, with the left side correlation coefficient of 0.60 and the right side correlation coefficient of 0.57 .

Conclusion There is a decrease in preclinical elasticity of carotid artery in patients with PLWH who take antiviral therapy for a long time, and ultrasound vascular elasticity quantitative technique is helpful to identify this change.

Keywords HIV; Atherosclerosis; Pulse wave velocity; Carotid intima-media thickness; Highly active 
antiretroviral therapy

\section{INTRODUCTION}

With the widespread use of HAART in many countries, the HIV/AIDS-related morbidity and mortality of PLWH have been significantly reduced. However, the special role of HIV and HAART drugs in promoting atherosclerosis significantly increases the risk of atherosclerotic cardiovascular disease (ASCVD) in PLWH $\left[{ }^{1,}\right.$ ${ }^{2]}$. ASCVD has become one of the important causes of PLWH's death. The development and application of non-invasive imaging technology to early predict PLWH atherosclerosis (AS) and guide clinical intervention has become a hot field of HIV research in recent years ${ }^{[3-5]}$. Compared with traditional ultrasonic morphological examination, ultrasonic vascular elasticity quantitative technique can reflect the changes of vascular elasticity before the formation of arterial plaques. The purpose of this study was to explore the feasibility of this technique in non-invasive evaluation of preclinical vascular elasticity in patients with atherosclerosis in PLWH population.

\section{Materials and Methods}

A total of 418 subjects, aged between 18 and 68 years were included in this study from November 2017 to February 2019.A retrospective study design was adopted and divided into baseline group, HAART group and healthy control group.

\section{Healthy control group}

60 healthy workers from the Third People's Hospital of Shenzhen from January 2019 to February 2019 were randomly selected, including 35 males and 25 females, aged (32.83 \pm 5.26$)$ years old.

\section{HIV baseline group}

291 newly diagnosed HIV/AIDS patients were randomly selected from November 2017 to February 2019 in Shenzhen third people's Hospital, including 272 males and 19 females, aged $33.29 \pm 9.73$ years. Diagnostic criteria for the asymptomatic phase ${ }^{[6]}$ : Epidemiological history, combined with HIV antibody positive can be diagnosed.The patients without clear epidemiological history but in line with laboratory diagnostic criteria can be diagnosed.None of the patients in this group received systematic HAART treatment before.

\section{HAART group}

Randomly selected 67 patients with HIV/AIDS who were treated in Shenzhen third people's Hospital from November 2018 to February 2019, including 54 males and 13 females, with an average age of (41.51 $\pm 8.95)$ years. This group of patients came from the eighth cohort of the national "13th five-year Plan" major science and technology project, and received standard HAART treatment.

All the subjects had no personal or family history of important organ diseases such as long-term smoking, alcoholism, hypertension, diabetes, coronary heart disease, heart disease, lung disease, nephropathy 
and so on.In addition, all the subjects had no long-term history of drug use that can easily lead to arterial disease.Blood glucose and blood pressure were normal, and body mass index ((BMI)) was within normal range. There were no abdominal and cardiac ultrasound abnormalities such as congenital heart disease, severe valvular heart disease, cardiomyopathy, cardiac insufficiency, space occupying lesions, renal artery stenosis and so on. Carotid ultrasound did not detect atherosclerotic plaque formation or arteritis. Carotid plaque is defined as ${ }^{[7]}$ : there is localized vascular wall thickening, at least $50 \%$ thicker than the surrounding vascular wall or IMT greater than $1.5 \mathrm{~mm}$, protruding into the vascular lumen and with a clear boundary with the surrounding vascular wall. All subjects had no found abnormal in chest x-ray and ECG. Pregnant women and subjects under 18 years old and over 70 years old were excluded from this study.

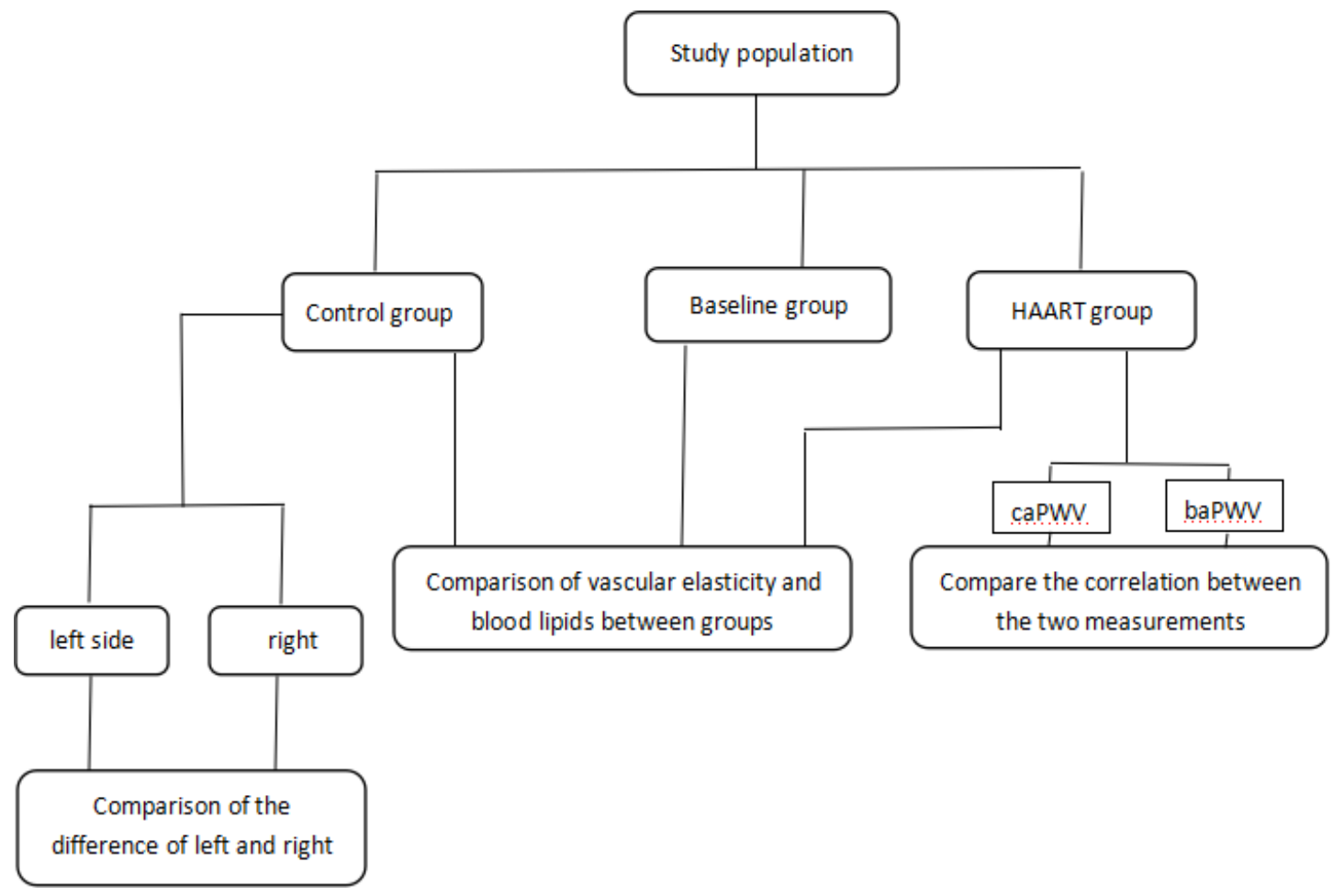

Figure 1 Study Group

\section{Statistical analysis}

SPSS25.0 software was used for statistical analysis, quantitative variables were expressed by mean \pm standard deviation $\left({ }^{\bar{x} \pm s}\right)$ in accordance with normal distribution, those who did not accord with normal distribution were expressed by median and quartile spacing [M (P25p75)], and qualitative variables were expressed by rate or constituent ratio. Comparison between groups: for quantitative variables, ANOVA was used for normal distribution, and Kruskal-Wallis $\mathrm{H}$ test was used for analyze unsatisfied variables. For qualitative variables, $\times 2$ test was used for disordered classification data, Bonferroni method was used for pairwise comparison, and the test level of pairwise comparison among the three groups was $\alpha=0.017$, with $\mathrm{P}<0.017$ as the difference, and the difference was statistically significant. Intra-group comparison: for quantitative variables, paired t-test was used for normal distribution, and symbolic rank sum test was used 
for dissatisfaction. For qualitative variables, paired $x 2$ test was used for disordered classification data, and the test level was $a=0.05$. The difference was statistically significant $(\mathrm{P}<0.05)$.

\section{RESULT}

One: the results of general situation comparison

There was no significant difference in height, weight, right systolic blood pressure, right diastolic blood pressure, left systolic blood pressure and left diastolic blood pressure between the baseline group and the HAART group.(Show Table 1)

Table 1: results of statistical analysis of various measurements in baseline group and HAART group

\begin{tabular}{cccc}
\hline Project & $\begin{array}{c}\text { Baseline group } \\
(\mathrm{n}=291)\end{array}$ & HAART group $(\mathrm{n}=67)$ & P value \\
\hline Height & $169.85 \pm 7.70$ & $168.64 \pm 7.39$ & 0.246 \\
Weight & $63.87 \pm 10.92$ & $62.04 \pm 9.35$ & 0.207 \\
Right systolic blood & $126.53 \pm 15.12$ & $126.45 \pm 17.99$ & 0.969 \\
$\begin{array}{c}\text { pressure } \\
\text { Right diastolic blood } \\
\text { pressure }\end{array}$ & $82.00 \pm 10.04$ & $81.61 \pm 11.76$ & 0.781 \\
$\begin{array}{c}\text { Left systolic blood pressure } \\
\text { Left diastolic blood } \\
\text { pressure }\end{array}$ & $124.38 \pm 16.63$ & $125.45 \pm 16.80$ & 0.638 \\
& $82.26 \pm 10.39$ & $82.33 \pm 11.12$ & 0.961 \\
\end{tabular}

Note: the baseline group is compared with the HAART group.

\section{Two: Comparison of ultrasonic elastic parameters}

Through the comparative analysis among the three groups, it was found that the bilateral carotid stiffness coefficient and caPWV in the HAART group were higher than those in the baseline group (P < 0.017), but the amplitude of motion in the HAART group was smaller than that in the baseline group, and the difference was statistically significant $(\mathrm{P}<0.017)$. TC, TG and HDL-C in HAART group were significantly higher than those in baseline group $(\mathrm{P}<0.017)$. (show table 2 and 3$)$.

Table 2: comparison of quantitative data of ultrasonic vascular elasticity of double carotid arteries among the three groups $(\bar{x} \pm s)$

\begin{tabular}{ccccc}
\hline $\begin{array}{c}\text { Elastic quantitative } \\
\text { data }\end{array}$ & $\begin{array}{c}\text { control group } \\
(\mathrm{n}=60)\end{array}$ & $\begin{array}{c}\text { Baseline group } \\
(\mathrm{n}=291)\end{array}$ & $\begin{array}{c}\text { HAART group } \\
(\mathrm{n}=67)\end{array}$ & P value \\
\hline $\begin{array}{c}\text { Right carotid artery } \\
\text { IMT }(\mathrm{mm})\end{array}$ & $0.66 \pm 0.12$ & $0.57 \pm 0.14$ & $0.71 \pm 0.14$ & $<0.001^{\text {ac }}$ \\
Right inner diameter & $6.62 \pm 0.56$ & $6.33 \pm 0.63$ & $6.29 \pm 0.68$ & 0.093 \\
\hline
\end{tabular}


$(\mathrm{mm})$

Right motion

$436.39 \pm 141.34$

$391.23 \pm 127.08$

$334.07 \pm 90.44$

$<0.001^{\text {bc }}$

amplitude (um)

Hardness coefficient

$3.26 \pm 0.92$

$3.40 \pm 1.29$

$3.93 \pm 1.04$

$0.005^{\mathrm{c}}$

on the right

Righ caPWV (m/s

$6.15 \pm 1.02$

$6.43 \pm 1.34$

$6.95 \pm 1.19$

$0.006^{\mathrm{c}}$

Left carotid artery

$0.66 \pm 0.11$

$0.56 \pm 0.13$

$0.72 \pm 0.17$

$<0.001^{\mathrm{ac}}$

IMT（ mm $)$

Left inner diameter

$6.30 \pm 1.02$

$6.20 \pm 0.62$

$6.19 \pm 0.82$

0.795

( $\mathrm{mm})$

Left motion

$396.78 \pm 82.14$

$386.46 \pm 129.74$

$320.30 \pm 99.92$

$<0.001^{\mathrm{c}}$

amplitude (um)

Hardness coefficient

$3.29 \pm 0.68$

$3.47 \pm 1.43$

$4.14 \pm 1.45$

$0.001^{\mathrm{c}}$

on the left

Left caPWV $(\mathrm{m} / \mathrm{s})$

$6.26 \pm 0.79$

$6.47 \pm 1.43$

$7.11 \pm 1.45$

$0.002^{\mathrm{c}}$

Note: IMT: intima-media thickness, Intima-Media Thickness;caPWV: pulse wave velocity, Pulse Wave Velocity a: healthy control group compared with baseline group, $\mathrm{P}<0.017$;b: healthy control group compared with HAART group, $\mathrm{P}<0.017$; c:baseline group compared with HAART group, $\mathrm{P}<0.017$.

Table 3: Comparison of blood lipid indexes between three groups $(\bar{x} \pm s)$

\begin{tabular}{ccccc}
\hline $\begin{array}{c}\text { Blood lipid } \\
\text { index }\end{array}$ & $\begin{array}{c}\text { Healthy control } \\
\text { group }(\mathrm{n}=60)\end{array}$ & $\begin{array}{c}\text { Baseline group } \\
(\mathrm{n}=291)\end{array}$ & $\begin{array}{c}\text { HAART } \\
(\mathrm{n}=67)\end{array}$ & group \\
\hline TC $(\mathrm{mmol} / \mathrm{l})$ & $4.72 \pm 0.83$ & $4.51 \pm 0.93$ & $4.92 \pm 1.17$ & $0.006^{\mathrm{c}}$ \\
$\mathrm{TG}(\mathrm{mmol} / \mathrm{l})$ & $1.28 \pm 0.62$ & $1.51 \pm 1.81$ & $2.45 \pm 2.43$ & $0.001^{\mathrm{c}}$ \\
$\mathrm{HDL}-\mathrm{C}(\mathrm{mmol} / \mathrm{l})$ & $1.34 \pm 0.25$ & $1.19 \pm 0.32$ & $1.33 \pm 0.34$ & $0.001^{\mathrm{c}}$ \\
LDL-C $(\mathrm{mmol} / \mathrm{l})$ & $2.82 \pm 0.64$ & $2.64 \pm 0.63$ & $2.77 \pm 0.72$ & 0.186 \\
\hline
\end{tabular}

Note: TC: Total Cholesterol; TG: triglyceride; HDL-C: high-density lipoprotein cholesterol; LDL-C:low-density lipoprotein cholesterol; a: healthy control group compared with baseline group, $\mathrm{P}<$ 0.017 , b: healthy control group compared with HAART group, $\mathrm{P}<0.017$. c: baseline group compared with HAART group, $\mathrm{P}<0.017$.

There was no significant difference between the left and right sides of the healthy control group $(\mathrm{P}>$ 0.05) (Show Table 4). 
Table 4:Comparison of quantitative data of ultrasonic vascular elasticity of bilateral carotid arteries in healthy control group $(\bar{x} \pm s)$

\begin{tabular}{llll}
\hline $\begin{array}{l}\text { Ultrasonic } \\
\text { quantitative data }\end{array}$ & \multicolumn{1}{c}{$\begin{array}{r}\text { elastic carotid artery } \\
(\mathrm{n}=23)\end{array}$} & $\begin{array}{c}\text { Right carotid artery } \\
(\mathrm{n}=23)\end{array}$ & P value \\
\hline Carotid artery IMT $(\mathrm{mm})$ & $0.66 \pm 0.11$ & $0.66 \pm 0.12$ & 0.988 \\
Inner diameter $(\mathrm{mm})$ & $6.30 \pm 1.02$ & $6.62 \pm 0.56$ & 0.107 \\
Range of motionum $)$ & $396.78 \pm 82.14$ & $436.39 \pm 141.34$ & 0.200 \\
Hardness coefficient & $3.29 \pm 0.68$ & $3.26 \pm 0.92$ & 0.853 \\
caPWV $(\mathrm{m} / \mathrm{s})$ & $6.26 \pm 0.79$ & $6.15 \pm 1.02$ & 0.577 \\
\hline
\end{tabular}

Note: IMT: Intima-Media Thickness; caPWV: Pulse Wave Velocity

Three:Correlation Analysis of caPWV and baPWV

Through the correlation analysis of caPWV and baPWV measured by ultrasound in HAART group, it was found that there was a linear correlation between RbaPWV and RPWV, the correlation coefficient was $0.588, \mathrm{P}<0.001$, the difference was statistically significant. There was a linear correlation between LbaPWV and LPWV, and the correlation coefficient was $0.565(\mathrm{P}<0.001)$. The difference was statistically significant.(Show figure 2)

Fig. 2 :correlation between caPWV and baPWV

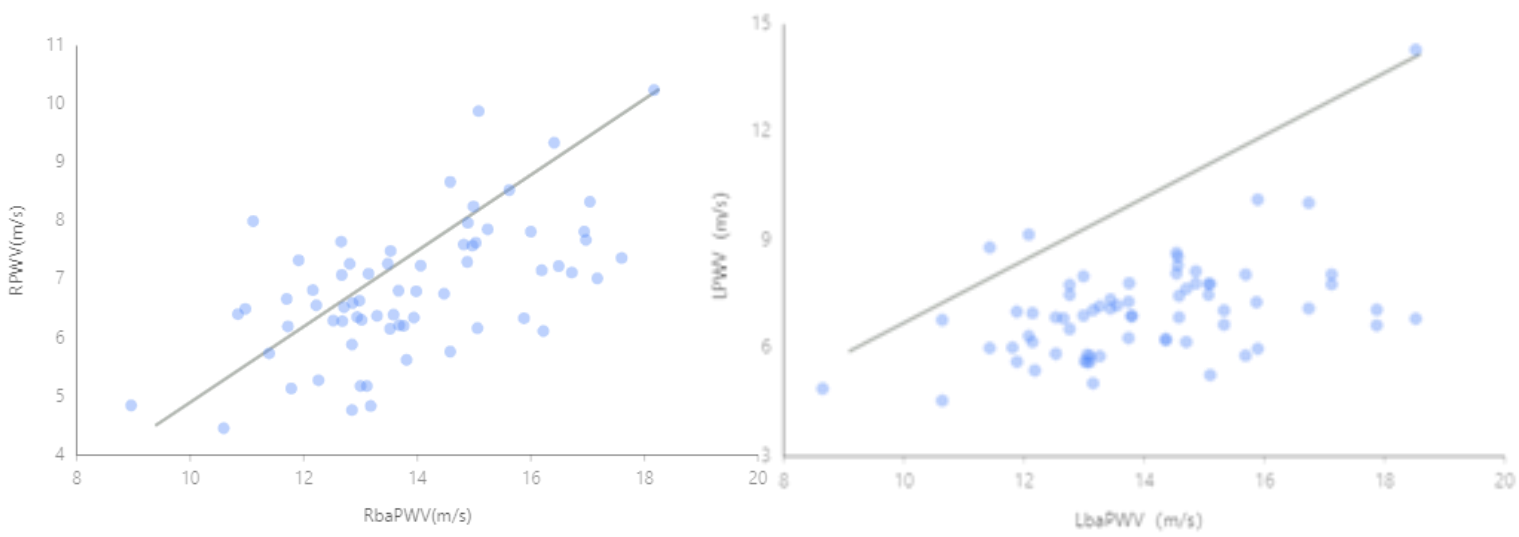

Note: caPWV: Pulse Wave Velocity; baPWV: Brachial ankle pulse wave velocity

\section{DISCUSSION}

Atherosclerosis is a chronic inflammatory disease that mainly affects the arterial intima and media, leading to smooth muscle cell proliferation and atherosclerosis. IMT thickness has a good correlation with 
atherosclerosis, and the use of ultrasound to measure the IMT thickness of the carotid artery to evaluate subclinical atherosclerosis has been widely accepted by the medical community. When IMT is greater than $0.9 \mathrm{~mm}$, ASCVD risk is higher ${ }^{[8]}$. This study found that the carotid IMT of PLWH who received HAART for more than 5 years was significantly thickened. Previous studies have also confirmed that the thickness of carotid IMT is related to long-term use of highly active antiretroviral therapy ${ }^{[9]}$, non-nucleoside reverse transcriptase inhibitors, and HIV Protein can induce arterial-related dysfunction ${ }^{[10]}$. HAART can cause endothelial cell activation in PLWH, inflammation, and carotid artery IMT increase. Inflammation markers and endothelial activation are all related to carotid artery IMT thickening.

Preclinical atherosclerosis refers to the presence of atherosclerosis in patients but the absence of severe stenosis of important arteries, leading to clinically clear symptoms of ischemia.In the early stages of the progression of arterial vascular disease, it is impossible to detect it with traditional imaging techniques. However, the increase in blood vessel diameter and the decrease in blood vessel elasticity may be reliable markers for atherosclerosis risk assessment ${ }^{[11]}$.The vascular motion amplitude, arterial stiffness coefficient, and PWV in the quantitative parameters of ultrasonic vascular elasticity can sensitively reflect the subtle changes in the carotid elastic function of PLWH.QAS technology plays an important role in the accurate analysis of arterial elasticity and can be used as a means of quantitative evaluation of early atherosclerosis ${ }^{[12]}$.None of the PLWH included in this study showed IMT thickening, but the ultrasound elasticity test results showed that the carotid artery stiffness coefficient and caPWV of PLWH who received HAART for more than 5 years increased significantly, the carotid artery motion amplitude decreased, and HAART treatment increased the arterial stiffness of the aorta Related ${ }^{[13]}$, decreased vascular elasticity may be related to the duration of HIV infection and long-term adverse effects of anti-HIV drugs. The results of this study showed that PLWH who received HAART for more than 5 years had significantly reduced carotid artery elasticity compared with new-onset PLWH who had never received HAART, which was consistent with previous studies.In addition, the motion amplitude of the right carotid artery of PLWH patients who had received HAART for more than 5 years was also lower than that of healthy people, while the motion amplitude of the right carotid artery showed a decreasing trend, although the comparison between the two was not statistically significant.

Anti-HIV drugs mainly include nucleoside reverse transcriptase inhibitors, non-nucleoside reverse transcriptase inhibitors, protease inhibitors and integrase inhibitors. HAART is a combination of three or more of these drugs.Some of them may cause adverse reactions such as high blood lipid and insulin resistance.Protease inhibitors represented by critazide are considered to have a higher risk of causing dyslipidemia and myocardial infarction, although they have good virological and immunological effects and a very good gene resistance barrier ${ }^{[14}{ }^{15]}$. This study found that the TC, TG, and HDL-c of PLWH who received HAART for more than 5 years were significantly increased. Previous studies have confirmed that HAART is closely related to hypercholesterolemia, the increase of TG and LDL-c and the decrease of HDL-c $\left.{ }^{[1617}\right]$.HAART can effectively inhibit the replication of HIV virus in PLWH, and significantly reduce 
the opportunistic infection and mortality of PLWH.On the one hand, clinical and experimental data show that inflammation plays an important role in atherosclerosi ${ }^{5[18]}$. However, HAART is less effective in eliminating HIV-related inflammation and innate immune activation. PLWH has a higher incidence of atherosclerosis than the general population.On the other hand, the side effects of antiretroviral drugs contribute to traditional CVD risk factors, including increased circulating levels of TC and TG, visceral obesity, and lower HDL-c levels $\left.{ }^{[19}\right]$.The mechanism of atherosclerosis caused by dyslipidemia is a hotspot of current research. HDL-C is one of the serum proteins that antagonize atherosclerosis,HDL-C carries the TC in the surrounding tissues, and then converts them into bile acids or is directly excreted from the intestine through bile.Studies have confirmed that there is a significant correlation between the decrease in HDL and the increase in the number of coronary artery stenosis in male patients ${ }^{[20]}$.In this study, HDL-c showed an increasing trend after HAART treatment, which was inconsistent with previous studies. Commonly used lipid-lowering therapies may be one of the reasons for this "abnormal phenomenon". The population in this study generally used ezetimibe , Atorvastatin combined with Probucol (E/A/P triple lipid-lowering), the effect of this therapy on HDL-c is significantly higher than that of $\mathrm{TC}$ and $\mathrm{TG}^{[21]}$.

In this study, it was found that there was no statistically significant difference in the quantitative data of bilateral carotid artery elasticity by ultrasound in the healthy control group.ASCVD as a systemic disease, the difference rate of detection methods in the carotid arteries on both sides should be as small as possible.The larger the difference rate is, it indicates that the local elasticity of the carotid artery measured by this method will be significantly different from that of other parts.Thus, it cannot truly reflect the degree of atherosclerosis of the whole body.PWV is the most important index used to quantify arterial elasticity. Carotid-femoral pulse wave velocity (CF-PWV) is considered as the gold standard for measuring $\mathrm{PWV}^{[22]}, \mathrm{cf}-\mathrm{PWV}$ is usually measured by using two sensors at two different locations on the body: one sensor is located on the skin above the carotid artery, and the other sensor is located above the femoral artery near the groin ${ }^{[23]}$.However, the test results are affected by the operator's experience, local pressure may also lead to patient discomfort, and the pressure reference point is difficult to unify.Mindray recently developed a technology called R-VQS (quantitative analysis of blood vessel stiffness based on rf data) that can measure changes in blood vessel diameter by analyzing radio frequency and accurately measuring the thickness of the vessel wall.This technology also combines systolic and diastolic blood pressure measurements, PWV and hardness coefficient calculations using reasonable formulas. The data generated by this technology reflects the elasticity of the blood vessel wall and provides valuable support for the early diagnosis of PLWH AS.The correlation analysis between the caPWV and baPWV of the HAART cases in this study showed that the correlation coefficient between the left caPWV and the left baPWV results was 0.6 , and the correlation coefficient between the right caPWV and the right baPWV results was $0.57(\mathrm{P}<0.05)$. The results of this study show that there is a certain correlation between this method and traditional methods, which indicates that this method is feasible in evaluating arterial elasticity, and the operation procedure is simpler and easier to be popularized. 


\section{Advantages and limitations of research}

Compared with previous studies, this study uses Mindray's newly developed R-VQS software to automatically and continuously measure the changes in the inner diameter of the blood vessel during six cardiac cycles through radio frequency data analysis and accurate tracking of the blood vessel wall. The measurement accuracy is high, and the measurement error is only $5 \mathrm{~mm}$. Through the measurement of systolic and diastolic blood pressure, the caPWV and the blood vessel stiffness coefficient are calculated through a reasonable formula, which accurately reflects the elasticity of the blood vessel wall. The sample size of this study is not enough, and further expansion and follow-up are needed. In addition, risk factors for atherosclerosis, such as HIV viral load, inflammation, and immune factors, that may affect the measurement of arterial elasticity between groups were not discussed.

\section{Future outlook}

Although most studies have shown that HIV, drugs, inflammation and immune response may be closely related to AS, there are few reports on the effects of different risk factors for AS by quantitative methods.In the future, this study will further quantify the impact of traditional AS factors and HIV-specific factors on AS in HIV populations.

\section{Conclusion}

Compared with healthy people, PLWH has reduced vascular motion amplitude, increased vascular stiffness coefficient and faster PWV. Ultrasound vascular elasticity technology is considered to be a viable tool for evaluating early changes in carotid atherosclerosis in AIDS patients and after HAART treatment.It is of positive significance for the early intervention and treatment of PLWH atherosclerosis and the prevention of cardiovascular and cerebrovascular diseases.

\section{Ethical statement}

This study was conducted in accordance with the ethical standards established by the Ethics Committee of Shenzhen Third People's Hospital, and was approved by the Committee. All subjects signed informed consent prior to participating in this study.

Fund

This research was funded by the Science and Technology Research Fund of Shenzhen (funded project JCYJ20200109144203815), and the National Natural Science Foundation of China (funded project 81971915), the Guangdong Medical Research Fund(funded project B20191163) and the National "Thirteenth Five-Year" Science and Technology Research Plan(funded project 2017ZX10202101-001-008).

\section{REFERENCES}

[1]Muller, E.V. and S. Gimeno, Risk factors for cardiovascular disease in HIV/AIDS patients treated with highly active antiretroviral therapy (HAART) in the central-southern region of the state of Parana - Brazil. Cien Saude 
Colet, 2019. 24(5): p. 1903-1914.

[2]Angelovich, T.A., et al., Monocytes from men living with HIV exhibit heightened atherogenic potential despite long-term viral suppression with antiretroviral therapy. AIDS, 2020. 34(4): p. 513-518.

[3]Papita, A., et al., Arterial stiffness and carotid intima-media thickness in HIV infected patients. Med Ultrason, 2011. 13(2): p. 127-34.

[4]Huang, C., et al., Non-Invasive Identification of Vulnerable Atherosclerotic Plaques Using Texture Analysis in Ultrasound Carotid Elastography: An In Vivo Feasibility Study Validated by Magnetic Resonance Imaging. Ultrasound Med Biol, 2017. 43(4): p. 817-830.

[]5 Liu, Z., et al., Interoperator Reproducibility of Carotid Elastography for Identification of Vulnerable Atherosclerotic Plaques. IEEE Trans Ultrason Ferroelectr Freq Control, 2019. 66(3): p. 505-516.

[6] Chinese guidelines for diagnosis and treatment of HIV/AIDS (2018)]. Zhonghua Nei Ke Za Zhi. 2018 Dec 1;57(12):867-884.

[7]Stein, J.H., C.E. Korcarz and W.S. Post, Use of carotid ultrasound to identify subclinical vascular disease and evaluate cardiovascular disease risk: summary and discussion of the American Society of Echocardiography consensus statement. Prev Cardiol, 2009. 12(1): p. 34-8.

[8]CENTURION O A. Carotid Intima-Media Thickness as a Cardiovascular Risk Factor and Imaging Pathway of Atherosclerosis[J]. Crit Pathw Cardiol, 2016,15(4): 152-160.

[9]LORENZ M W, STEPHAN C, HARMJANZ A, et al. Both long-term HIV infection and highly active antiretroviral therapy are independent risk factors for early carotid atherosclerosis[J]. Atherosclerosis, 2008,196(2): 720-726.

[10]HANSEN L, PARKER I, SUTLIFF R L, et al. Endothelial dysfunction, arterial stiffening, and intima-media thickening in large arteries from HIV-1 transgenic mice[J]. Ann Biomed Eng, 2013,41(4): 682-693.

[11]YOSHINAGA K, FUJII S, TOMIYAMA Y, et al. Anatomical and Functional Estimations of Brachial Artery Diameter and Elasticity Using Oscillometric Measurements with a Quantitative Approach[J]. Pulse (Basel), 2016,4(1): 1-10.

[12]HUANG X, KANG X, XUE J, et al. Evaluation of carotid artery elasticity changes in patients with cerebral small vessel disease[J]. Int J Clin Exp Med, 2015,8(10): 18825-18830.

[13]van VONDEREN M G, SMULDERS Y M, STEHOUWER C D, et al. Carotid intima-media thickness and arterial stiffness in HIV-infected patients: the role of HIV, antiretroviral therapy, and lipodystrophy[J]. J Acquir Immune Defic Syndr, 2009,50(2): 153-161.

[14]KOPPEL K, BRATT G, ERIKSSON M, et al. Serum lipid levels associated with increased risk for cardiovascular disease is associated with highly active antiretroviral therapy (HAART) in HIV-1 infection[J]. Int J STD AIDS, 2000,11(7): 451-455.

[15]OLCZAK A. [Metabolic disturbances associated with HAART][J]. Przegl Epidemiol, 2007,61(4): 639-646.

[16]NSAGHA D S, WELEDJI E P, ASSOB N J, et al. Highly active antiretroviral therapy and dyslipidemia in people living with HIV/AIDS in Fako Division, South West Region of Cameroon[J]. BMC Cardiovasc Disord, 2015,15: 95.

[17]GIANNARELLI C, KLEIN R S, BADIMON J J. Cardiovascular implications of HIV-induced dyslipidemia[J]. Atherosclerosis, 2011,219(2): 384-389.

[18]MOREIRA D M, DA S R, VIEIRA J L, et al. Role of vascular inflammation in coronary artery disease: potential of anti-inflammatory drugs in the prevention of atherothrombosis. Inflammation and anti-inflammatory drugs in coronary artery disease[J]. Am J Cardiovasc Drugs, 2015,15(1): 1-11.

[19] ANGELOVICH T A, TREVILlYAN J M, HOY J F, et al. Monocytes from men living with HIV exhibit heightened atherogenic potential despite long-term viral suppression with antiretroviral therapy[J]. AIDS, 2020,34(4): 513-518. 
[20] SONG Y, YANG Y, ZHANG J, et al. The apoB100/apoAI ratio is independently associated with the severity of coronary heart disease: a cross sectional study in patients undergoing coronary angiography[J]. Lipids Health Dis, 2015,14: 150.

[21] GUO Y S, WANG C X, CAO J, et al. Antioxidant and lipid-regulating effects of probucol combined with atorvastatin in patients with acute coronary syndrome[J]. J Thorac Dis, 2015,7(3): 368-375.

[22]Stone NJ, Robinson JG, Lichtenstein AH, et al.2013 ACC/AHA guideline on the treatment of blood cholesterol to reduce atherosclerotic cardiovascular risk in adults: a report of the American College of Cardiology/American Heart Association Task Force on Practice Guidelines. J Am Coll Cardiol, 2014. 63(25 Pt B): p. 2889-934.

[23] LI Y, SEGERS P, DIRCKX J, et al. On-chip laser Doppler vibrometer for arterial pulse wave velocity measurement[J]. Biomed Opt Express, 2013,4(7): 1229-1235. 